\title{
Benefits and Barriers of ICU Daily Round; Perspective of Intensivists: Cross-Sectional Study
}

\author{
Ahmed F. Mady ${ }^{1,2}$, Ahmed Elalfy ${ }^{1}$, Mostafa Y. Elawadi ${ }^{1}$, Emad I. Sawaby ${ }^{1}$, \\ Sayed S. Ahmad ${ }^{1}$, Ihab A. Nassar', Ayman S. Abo-khoud ${ }^{1}$, \\ Mostafa S. Mahmoud ${ }^{1}$, Hamdy M. Abdulgawwad ${ }^{1}$, Hamdy M. Rashid ${ }^{1}$, \\ Hany A. Asaker ${ }^{1}$, Waleed T. Aletreby ${ }^{1}$, Khalid Y. Eldar ${ }^{1}$, \\ Mohammed Abdulazim¹, Mostafa M. Selem¹, Kriz L. Odchigue ${ }^{1}$ \\ ${ }^{1}$ Critical Care Department, King Saud Medical City, Riyadh, Saudi Arabia. \\ ${ }^{2}$ Anesthesia Department, Faculty of Medicine, Tanta University, Tanta, Egypt.
}

Corresponding Author: Waleed T. Aletreby

DOI: https://doi.org/10.52403/ijhsr.20220202

\begin{abstract}
Daily ICU round has become the standard of care in the intensive care unit. It has varying structures and designs across ICUs world-wide.

Aim: Cross-sectional study to explore the perspective of ICU physicians regarding the daily round.

Method: Online survey including questions in four domains, namely, physicians' gain, patients' gain, barriers to the round, and suggestions for improvement.

Results: Perceived physicians' gains in order were: role clarity (73\%), newly obtained knowledge $(61 \%)$, medico-legal protection (45\%), and communication with other disciplines (30\%). As for patients' gain, the perceived benefits were: making intervention decisions (97\%), objectives of management $(81 \%)$, better patients' outcomes $(55 \%)$, and a tool to respond to patients' conditions $(15 \%)$. Barriers to the round were lack of standardization and input from other disciplines, long duration and interruptions. The physicians suggested a standardized structured tool for the round as a method of improvement.

Conclusion: ICU physicians' main perceived personal gain from the daily ICU round is a formulated plan of management, for patients' gain is decision making, as a barrier is the lack of standardization, and suggest its standardization for improvement.
\end{abstract}

Keywords: ICU physicians, ICU daily round, Intensivists, intensive care unit

\section{INTRODUCTION}

Since its publication in 2000, the landmark report by the Institute of Medicine "To Err is Human" (1) has led to numerous and comprehensive reforms in the structure of healthcare systems. One of the major recommendations of the report, was the provision of safe healthcare. Accordingly, multidisciplinary rounds in intensive care units (ICU) became a standard of care, since it is considered as the mainstay of communication among different teams involved in the management of patients (2), and up to $70 \%$ of errors are results of miscommunication (3).

Several studies have demonstrated the benefits of daily multidisciplinary rounds, with regards to different outcomes, such as mortality (4), adherence to protocols (5) and length of stay (LOS) (6). However, despite the undeniable benefits of daily rounds in ICU, the structure of the round itself remains a subject of significant variations and differences between institutes, as well as units within the same institute (7) regarding many aspects of the 

study.

rounds' structure such as involved members, sources of input, and tasks to be fulfilled during the round $(2,8,9)$.

Many studies, with different designs investigated the daily round with regards to patients' outcomes, providers' satisfaction, or the impact of a certain intervention on measurable outcomes $(6,9,10)$. However, few studies explored the perspective of intensivists and bed-side physicians of the daily round. We conducted this crosssectional study to enlighten about how ICU physicians perceive the ICU daily round, its benefits, barriers, and suggestions for improvement.

\section{METHOD}

This was a cross-sectional study in an electronic survey format conducted in the adult ICU of King Saud Medical City (KSMC) between December 1 and December 31, 2021. The ICU harbors 127 beds, divided in seven units (Trauma, Respiratory, Neuro-critical care, Medical, Surgical, Burn, and Maternity) The ICU employs 40 specialists and 45 residents, in addition to 30 physicians in rotation from the Saudi Critical Care Board. The study was approved by the local institutional review board, considering responding to the survey as the consent of the physician to participate in the study.

\section{Outcomes}

Questions of the survey were structured to capture the opinion of ICU physicians about the daily round with regards to four categories: Personal gain (focusing on benefits attained by the round for the physicians themselves), patients' gain (perceived benefits for the patients), barriers to ideal round, and an open end question of suggestions to improve the round. In addition to demographic data of participants (age, gender, ICU experience).

\section{The Survey}

Google forms freely available service was used to create the survey. The questions were validated by three authors
(W. A., A. A., and M. Y.) for clarity of the questions, duration to complete the survey, and ability to capture the intended objectives, by examining the responses of a pilot sample of 10 respondents. Apart from the demographic questions, each question in the survey could be answered on a 5-pointLikert scale, Link to the survey is available at: (https://forms.gle/fQ3YEf7ckcKk8qsT6)

We invited participation through emails to ICU physicians, followed by reminder emails after one week. We also posted an invitation to participate as well as the link to the survey on social media platforms of the ICU.

\section{Inclusion and exclusion criteria}

We included ICU physicians whether they were specialists, residents, or on rotation, provided they have experience with the daily ICU round with at least three different consultants. We excluded ICU consultants (as they are the ones conducting the round), and other members of the multidisciplinary team (such as nurses, clinical pharmacists, and respiratory therapists) since other studies were being conducted simultaneously in our ICU involving those categories with similar objectives.

\section{Sample size}

The ICU includes a total of 115 physicians (in different categories) to whom the survey is intended. In order to have results with $95 \%$ confidence interval and $5 \%$ margin of error, we required 89 respondents to our survey.

\section{Data analysis and statistical considerations}

Categorical demographic data and responses on the Likert scale were presented as frequency and percentage. We manually summarized the responses to "Suggestions of improvement", grouped similar suggestions together, and presented the top five suggestions.

We explored whether the overall score of any of the two categories "Personal gain" and "Patients' gain" was correlated to 
Ahmed F. Mady et.al. Benefits and barriers of ICU daily round; perspective of intensivists: cross-sectional study.

any demographic criteria of respondents, utilizing the non-parametric Kruskal-Wallis test, at a significant level of $5 \%$, post hoc pairwise comparisons according to Conover method (11), and test of trends for ordered categories. To carry out Kruskal-Wallis test, we assigned a value from 1-5 (1 being the most negative response and 5 the most positive) to each response on the Likert scale, the sum of the values by each respondent in the categories of "Personal gain" and "patients' gain" give the overall score for the category.

\section{RESULTS}

Table 1: Demographic data of respondents:

\begin{tabular}{|l|l|}
\hline Attribute $(\mathbf{n = 9 7})$ & Number $(\%)$ \\
\hline Gender: & $89(91.7 \%)$ \\
Males & $8(8.3 \%)$ \\
Females & \\
\hline Age group: & $37(38.1 \%)$ \\
$18-30$ years & $15(15.5 \%)$ \\
$30-40$ years & $40(41.2 \%)$ \\
$40-50$ years & $5(5.2 \%)$ \\
More than 50 years & \\
\hline ICU Position: & $29(30 \%)$ \\
Rotation & $30(31 \%)$ \\
Resident & $38(39 \%)$ \\
Specialist & \\
\hline ICU Experience: & $29(29.8 \%)$ \\
Less than 2 years & $19(19.6 \%)$ \\
$2-5$ years & $44(45.4 \%)$ \\
$5-10$ years & $5(5.2 \%)$ \\
More than 10 years &
\end{tabular}

Table 2: Analysis of variance of "personal gain" and "patients' gain" scores, across categories of: Age, ICU experience, ICU position, and gender:

\begin{tabular}{|l|l|l|l|l|l|l|}
\hline \multirow{2}{*}{ Categorical Factor } & \multicolumn{2}{|l|}{ Kruskal-Wallis test } & \multicolumn{2}{l|}{ Post hoc pairwise comparison* } & \multicolumn{2}{l|}{ Trend Test } \\
\cline { 2 - 7 } & Personal gain & Patients' gain & Personal gain & Patients' gain & Personal gain & Patients' gain \\
\hline Age Categories & 0.03 & 0.002 & $\begin{array}{l}\text { B \& A } \\
\text { B \& C }\end{array}$ & $\begin{array}{l}\text { D \& A } \\
\text { D \& B } \\
\text { D \& C }\end{array}$ & 0.8 & 0.2 \\
\hline Experience Categories & 0.02 & 0.002 & $\begin{array}{l}\text { B \& A } \\
\text { B \& C }\end{array}$ & $\begin{array}{l}\text { D \& A } \\
\text { D \& B } \\
\text { D \& C }\end{array}$ & 0.97 & 0.4 \\
\hline Position Categories & 0.06 & 0.09 & -------------- & -------------- & 0.9 & 0.1 \\
\hline Gender Categories & 0.002 & 0.004 & A \& B & A \& B & 0.002 & 0.005 \\
\hline
\end{tabular}

Age (years): $A=18-30, B=30-40, C=40-50, D=$ More than 50

Experience (years): $A=$ less than 2, $B=2-5, C=5-10, D=$ more than 10

Position: $A=$ Rotation, $B=$ Resident,$C=$ Specialist

Gender: $A=$ Female, $B=$ Male

$* P<0.05$ for each pair.

In the category of "Patients' gain" an overwhelming majority of $97 \%(95 \% \mathrm{CI}$ : $91.4-99.4 \%$ ) believed that the daily round was beneficial in taking decisions about interventions, whether invasive or radiological to be carried out. Next, was the believe that clear objectives of the management plan were decided in the round, as $81 \%(95 \% \mathrm{CI}: 71.8-88.3 \%)$ indicated the clarity of objective to be (Very Much) or (Much). Slightly above half of the respondents thought that the daily round results in better patients' outcomes, 35\% (95\% CI: $25.6-45.5 \%)$ were (Neutral), and $10 \%$ (95\% CI: $4.8-17.8 \%$ ) thought that the round resulted in (Little) or (Very Little) 

study.

better patients' outcomes. However, most of the respondents $(85 \% \quad(95 \%$ CI: 76.3 91.4\%)) believed that the round was (Little) or (Very Little) helpful as a tool to respond to dynamic patients' conditions (Figure 2).

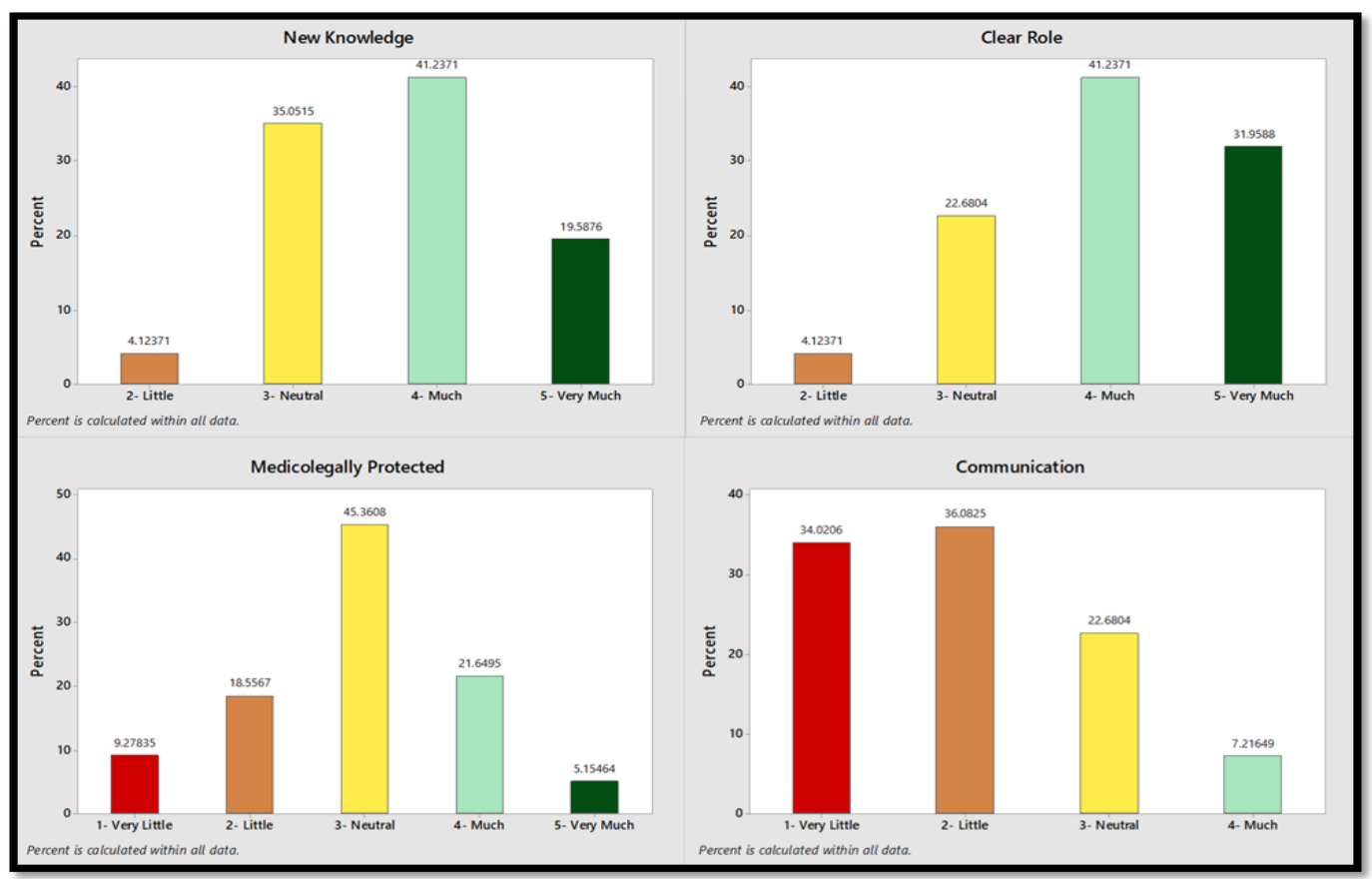

Figure 1: Responses in the category of "Personal gain":

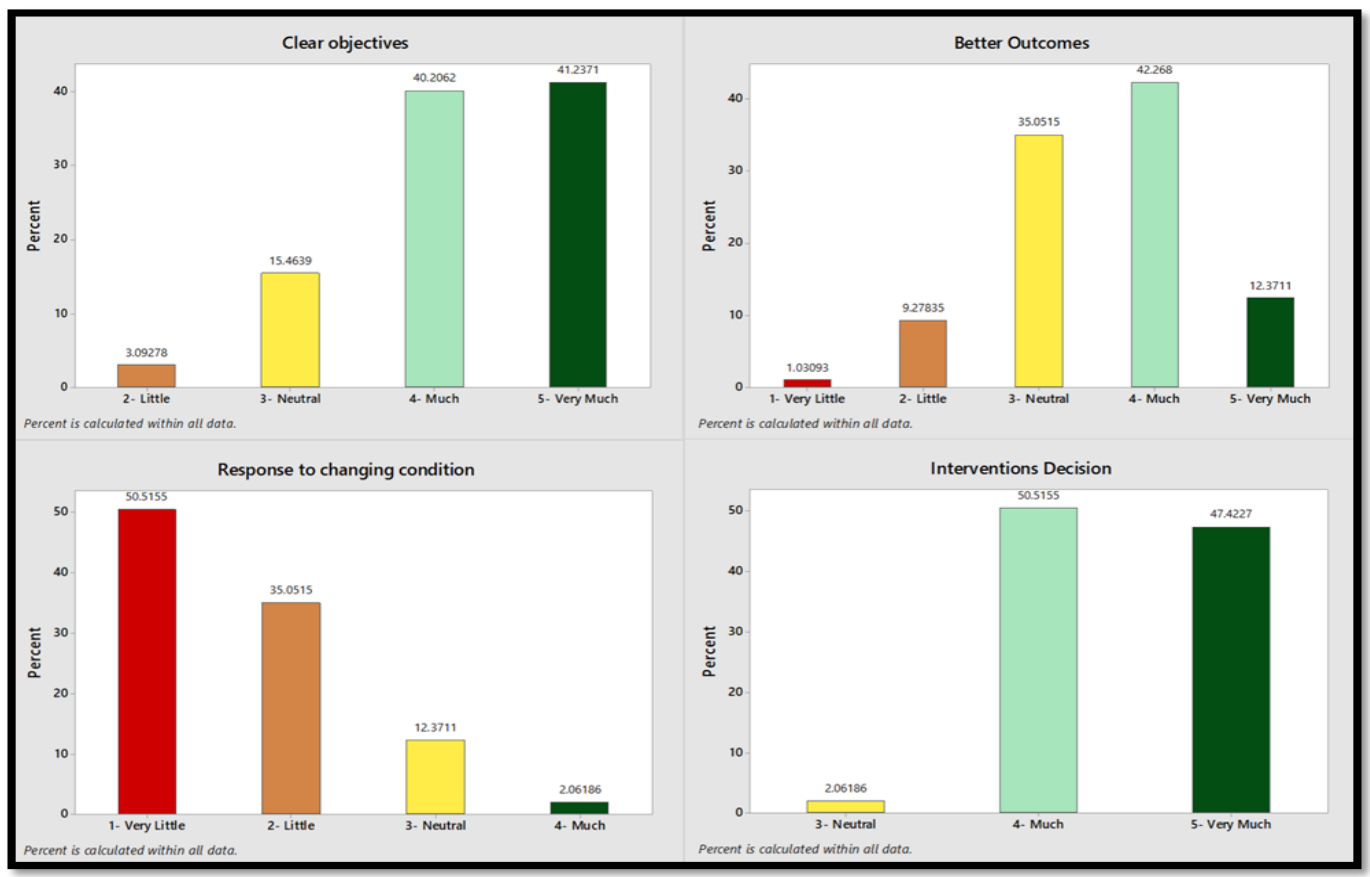

Figure 2: Responses in the category of "Patients' gain":

In the category of "Barriers" most of the responses were on the negative side, as about $80 \%$ (95\% CI: $70.7-87.4 \%$ ) thought that the round lacked a standardized structure, with only $20 \%$ disagreeing. With regards to input by other disciplines, $90 \%$ (95\% CI: $82.2-95.2 \%$ ) agreed or strongly agreed that the round lacked input by other specialties, no one disagreed. More than half of the respondents thought that the duration of the round was adequate, the remaining responses were on the (Long) and (Too Long) side, no one thought the round was shorter than it should be. And finally, with 
Ahmed F. Mady et.al. Benefits and barriers of ICU daily round; perspective of intensivists: cross-sectional study.

regards to interruption, most of responses were (Neutral), with slightly more perceiving (Much) or (Very Much) interruptions than those perceiving (Little) interruption (Figure 3).

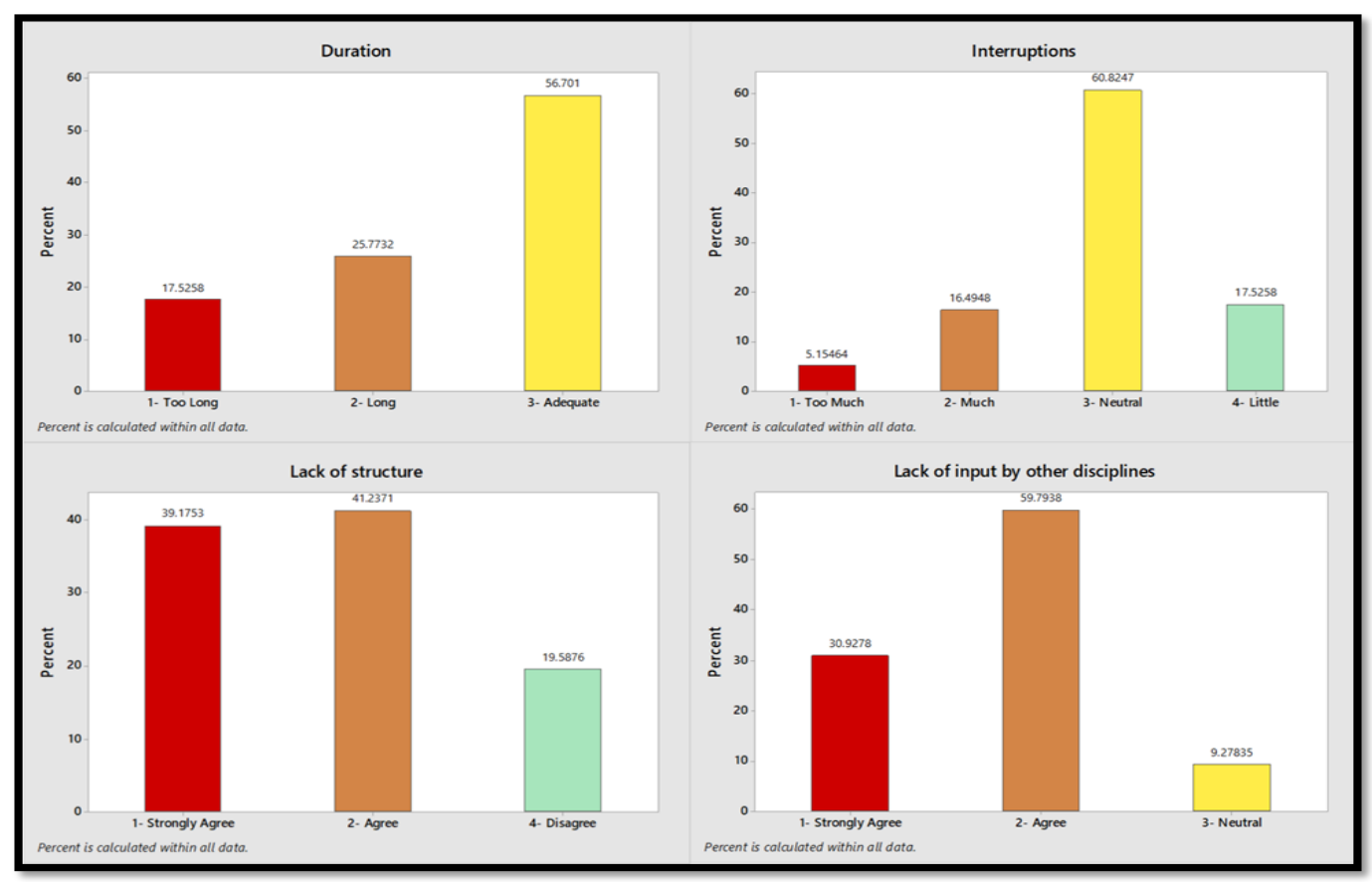

Figure 3: Responses in the category of "Barriers to proper round":

The most frequent round improvement suggestion was to have a standardized structure for the round, details for that suggestion included a checklist or predefined items to go through during the round. Then came the suggestion that more input from all members of the team should be sought after, another suggestion took it a step further, and suggested different aspects of the patient's condition to be the responsibility of the relevant member of the healthcare team, for example the vital signs to be endorsed by the nurse, the ventilator settings and oxygenation status by the respiratory therapist, the medications by the clinical pharmacist, and so on. Another frequent suggestion was to assign a junior physician (other than the responsible bedside) to paperwork duties during the round, such as documenting the consultant's plan, or modification of medications. Some respondents suggested scheduling the ICU round with the round of the primary admitting team as a way of improving communication and coordinating the plan of management. In our ICU the consultants are assigned to unit of the ICU and this assignment changes weekly, one suggestion to improve the round was to require the same ICU consultant to continue to care for the patient who was admitted during his/her on call, regardless of the unit, this is to avoid wasting time to orient the consultant at the beginning of the week with the patients in the unit, and as a better way to accountability and continuity of care.

Table 2 shows details of KruskalWallis test. In the category of "Personal gain", age group $(30-40)$ had a higher median score compared to age groups (18$30)$ and $(40-50)$, but not to (more than 50$)$. The experience category of $(2-5)$ years had a higher median score compared to (Less than 2 years) and $(5-10)$ years, but not different from (More than 10 years), males had a higher median score compared to females. There was no difference between categories of ICU position.

For the category of "Patients' gain", the age group (More than 50) had a higher median score compared to all other groups, and the group with more than 10 years of 

study.

experience had a higher median score than all other groups. There was no difference between groups of ICU position, and males had a higher median score compared to females.

All tests of trends were statistically insignificant, apart from gender which may not be informative due gender imbalance in our sample.

\section{DISCUSSION}

Our study shows that ICU physicians perceive clarity of their role as the most important personal gain from the daily round, while communication with other teams as the least perceived gain. The groups with highest scores in that category were physicians between 30 and 40 years old, and with 2 to 5 years of experience. As for the patients' gain, the most perceived gain was making decisions about interventions. The least perceived gain for the patients was responding to changing medical condition. In that category, the highest scores were from physicians older than 50 years old, and with more than 10 years of experience. The ICU physicians perceived the daily round as lacking a structure, missing input from various team members, longer than it should be, and frequently interrupted. Accordingly, they suggested having a structured round, with required input from all healthcare providers, minimization of paper and administrative work, and the same consultant to continue to care for the same patients without rotation.

The daily ICU round has become a standard of care, and a tool to formulate management plans and objectives (9), however; few studies examined the way ICU physicians see the round. The results of our survey seem to reflect a view of ICU physicians that is mostly consistent with findings of others. In our study, the most perceived personal gain was clarity of role, while the highest perceived patients' gain was decision making about interventions, this is echoed in other studies where $72 \%$ of responses stated that the purpose of the round is formulating the plan of care and setting management goals (2), in the same study, only $11 \%$ believed that the round was a teaching tool, however; a higher percentage $(61 \%)$ in our study thought so, probably because $30 \%$ of our sample size weren't service physicians, but fellows or residents in training programs. Perhaps it's understandable why the ICU physicians felt neutral about the round as a protective mechanism, in view of accountability practices in almost all healthcare systems. Similarly, a high percentage of perceived better patients' outcomes is comprehendible, particularly among the more experienced physicians, which was objectively measured by others (11). Low ratings of perceived communication in our survey were also shown by others (12), as this issue seems to be the most frequent obstacle to an effective round. Whereas; the ever changing dynamic status of ICU patients may be the reason behind similarly low ratings of viewing the round as a tool to responding to such changing conditions.

In the domain of barriers, the findings of our survey were aligned with those reported by many other studies, such as frequent interruptions identified as one of the major barriers to the daily round in a systematic literature review (13), in the study by O'Brien et al (9) there was a poor input by other disciplines participating in the management of patients, and that participation could be improved by standardizing the structure of the round. Both elements (lack of input and standardization) were identified by our responders as barriers.

ICU physicians suggested standardization of the daily round as an improvement tool, second was the suggestion aimed at increasing input and participation by different team members in the form assigning each member certain responsibilities to be endorsed, their suggestions also included different ways to help the ICU physician doing the round to focus on the patient, by delegating other administrative duties and paperwork to other team members. All those suggestions 
Ahmed F. Mady et.al. Benefits and barriers of ICU daily round; perspective of intensivists: cross-sectional study.

appeared previously in the scarce perspective studies, as well as studies describing the structure of an ICU round (2, 7,9).

Our correlational analysis shows that younger less experienced physicians perceive higher benefits for the physician from the daily round, this could mean that such perceived benefits may be less evident for older physicians with more experience, indicating that those benefits may not actually be realistic. The opposite is seen for the patients' gain, the older experienced physicians scored higher in this domain, possibly meaning that this is an opinion they gained from experience.

We present the results of this survey to administrative and policy makers in our institute, to carefully examine and perhaps act upon, in order to improve the daily ICU round.

This study suffers numerous limitations. It shows the opinion of physicians from a single center, so reflecting characteristics of the round specific for our center. The sample size in our study is small, and in order to have a clearer picture of ICU perception of the daily round a much larger sample size is required. Our survey was mostly closed end questions, apart for the suggestions of improvement section, and it is quite possible that we missed certain aspects of the round that physicians may have in mind. Our correlational analysis was not powered to detect correlation, accordingly; its results must be interpreted with caution.

\section{CONCLUSION}

ICU physicians' main perceived personal gain from the daily ICU round is a formulated plan of management, for patients' gain is decision making, as a barrier is the lack of standardization, and suggest its standardization for improvement.

\section{Conflict of Interest Statement}

All authors declare no conflict of interest.

\section{Funding Statement}

No personal or institutional funding

was received for this study.

\section{Acknowledgement: None}

\section{Ethical Approval: Approved}

\section{REFERENCES}

1. Institute of Medicine (US) Committee on Quality of Health Care in America. To Err is Human: Building a Safer Health System. Kohn LT, Corrigan JM, Donaldson MS, editors. Washington (DC): National Academies Press (US); 2000.

2. Giri, Jyothsna, Adil Ahmed, Yue Dong, Mark Keegan, Vitaly Herasevich, Brian W. Pickering and Ognen Gajic. "Daily Intensive Care Unit Rounds: A Multidisciplinary perspective." Applied Medical Informatics 33 (2013): 63-73.

3. H. Singh, E.J. Thomas, L.A. Petersen, D.M. Studdert. Medical errors involving trainees: a study of closed malpractice claims from 5 insurers. ArchInternMed 2007;167(19):2030 2036

4. Vats A, Goin KH, Villarreal MC, Yilmaz T, Fortenberry JD, Keskinocak P. The impact of a lean rounding process in a pediatric intensive care unit. Critical care medicine. [Research Support, Non-U.S. Gov't]. 2012;40(2):608-17.

5. B.S. Cypress. Family presence on rounds: a systematic review of literature. Dimensions of critical care nursing: DCCN 2012;31(1):53-64.

6. Kim MM, Barnato AE, Angus DC, Fleisher LA, Kahn JM. The effect of multidisciplinary care teams on intensive care unit mortality. Arch Intern Med. 2010 Feb 22;170(4):369-76. doi: 10.1001/archinternmed.2009.521. Erratum in: Arch Intern Med. 2010 May 24;170(10):867.

7. Amaral ACKB, Vincent JL, Rose L, Mikkelsen ME, Webb S, Ramos GV, Pinto $\mathrm{R}$, Salluh J. An international perspective on the frequency, perception of utility, and quality of interprofessional rounds practices in intensive care units. J Crit Care. 2020 Feb;55:28-34.

8. J.K. Holodinsky, M.A. Hebert, D.A. Zygun, R. Rigal, S. Berthelot, D.J. Cook, et al. A Survey of Rounding Practices in Canadian 
Ahmed F. Mady et.al. Benefits and barriers of ICU daily round; perspective of intensivists: cross-sectional study.

Adult Intensive Care Units. PloS one 2015;10(12):e0145408.

9. O'Brien A, O'Reilly K, Dechen T, Demosthenes N, Kelly V, Mackinson L, Corey J, Zieja K, Stevens JP, Cocchi MN. Redesigning Rounds in the ICU: Standardizing Key Elements Improves Interdisciplinary Communication. Jt Comm J Qual Patient Saf. 2018 Oct;44(10):590598.

10. Paradis E, Leslie M, Gropper MA. Interprofessional rhetoric and operational realities: an ethnographic study of rounds in four intensive care units. Adv Health Sci Educ Theory Pract. 2016 Oct;21(4):735-48.

11. Wheelan SA, Burchill CN, Tilin F. The link between teamwork and patients' outcomes in intensive care units. Am J Crit Care. 2003 Nov;12(6):527-34.
12. Cao V, Tan LD, Horn F, Bland D, Giri P, Maken K, Cho N, Scott L, Dinh VA, Hidalgo D, Nguyen HB. Patient-Centered Structured Interdisciplinary Bedside Rounds in the Medical ICU. Crit Care Med. 2018 Jan;46(1):85-92.

13. Gurses AP, Xiao Y. A systematic review of the literature on multidisciplinary rounds to design information technology. J Am Med Inform Assoc. 2006 May-Jun;13(3):267-76.

How to cite this article: Ahmed F. Mady, Ahmed Elalfy, Mostafa Y. Elawadi et.al. Benefits and barriers of ICU daily round; perspective of intensivists: cross-sectional study. Int J Health Sci Res. 2022; 12(2): 8-15. DOI: https://doi.org/10.52403/ijhsr.20220202 